\title{
Estimation of Lower Jaw Density using CT data
}

\author{
Ariunbold Jargalsaikhan ${ }^{1}$, Nyamlkhagva Sengee ${ }^{1 *}$, Berekjan Telue $^{1}$, Sambuu Ochirkhvv $^{1}$
}

\begin{abstract}
Bone density is one of the factors in the early failure of dental implants and doctors should make a preoperative assessment of jaw bone density using patient's CT data before dental implant surgery in order to find out whether the patient has osteoporosis and osteopenia. The main goal of this study was to propose a method that based on image processing techniques in order to provide accurate information about where to drill and place an abutment screw of implants in the jaw bone for doctors and reduce human activity for the estimation of the local cancellous bone density of mandible using CT data. The experiment was performed on a computed tomography data of the jaw bone of two different individuals. We assumed that the result of the estimation of jaw bone density depends on the angle of drilling and average HU (Hounsfield Unit) values were used to evaluate the quality of local cancellous bone density of mandible. As a result of this study, we have been developed a toolbox that can be used to estimate jaw bone density automatically and found a positive correlation between the angle of the drill and time complexity but a negative correlation between the diameter of the drill and time complexity.
\end{abstract}

Key Words: Computed tomography (CT), mandibular density, lower jaw, medical image processing

\section{INTRODUCTION}

Nowadays, the dental implants that are surgically embedded into the jaw and fuse with the bone over 3-6 months depending on the situation have become the preferred way to recreate teeth and the global dental implants market size growing at CAGR (Compound Annual Growth Rate) of 7.7\% over the forecast period [1]. In addition, according to the American Academy for Implant Dentistry, in the U.S., over 15 million people per year undergo bridge and crown replacements for missing teeth. Hence, facilitating extensive demand for dental implants.

The success rate of dental implants has been reported in scientific literature as 98 percent [2] and jaw bone density is one of the factors in the early failure of dental implants [3]. Therefore, doctors should make a preoperative assessment of jaw bone density using the patient's CT data before dental implant surgery in order to find out whether the patient has osteoporosis and osteopenia. Hence, in this paper, we presented a method based on image processing techniques in order to reduce human activity for the estimation of the local cancellous bone density of mandible and provide accurate information about where to drill and place an abutment screw of implants in the jaw bone for doctors using patient's CT data.
In the study, the lower jaw was used that manually extracted from the computer tomography data, because of placing implants into the upper jaw is riskier than the lower jaw. Also, local bone density means that calculate for a tooth that selected from CT data by the doctor who is going to perform the procedure.

The experiment was performed on a computed tomography data of the jaw bone of two different individuals. We assumed that the result of the estimation of jaw bone density depends on the angle of drilling and average HU (Hounsfield's Unit) values were used to evaluate the quality of local cancellous bone density of mandible.

As a result of this study, we have been developed a toolbox that can be used to estimate jaw bone density automatically and found a positive correlation between the angle of the drill and time complexity but a negative correlation between the diameter of the drill and time complexity. Also, the toolbox can be used for planning surgical procedures of dental implants.

In what follows, related works are discussed in Section 2 and Section 3 presents the proposed method and its improvement. Section 4 shows the experimental results of the study. Section 5 serves as the conclusion and future work of the paper.

\footnotetext{
Manuscript received April 29, 2019; Revised May 19; Accepted May 21, 2019. (ID No. JMIS-19M-04-015) Corresponding Author $\left(^{*}\right)$ : Nyamlkhagva Sengee, IKH SURGUULIIN GUDAMJ-1 P.O.BOX -46A/523, 210646, nyamlkhagva@seas.num.edu.mn

${ }^{1}$ National University of Mongolia, ULAANBAATAR, MONGOLIA, ariukajagaa@gmail.com, d.se11d104@gmail.com, sambuuochirkhvv@yahoo.com
} 


\section{RELATED WORKS}

Computed tomography (CT) is usually available in emergency units, and CT scans of proximal humerus fractures are frequently performed in order to precisely classify the fracture and to plan medical procedures. There are some research work about Hounsfield Unit (HU) based standardized method for the preoperative assessment of the cancellous bone mineral density (BMD) of the humeral head using CT data [4].

Assessing local bone quality on CT scans with Hounsfield unit (HU) quantification is being used with increasing frequency. Correlations between $\mathrm{HU}$ and bone mineral density have been established, and normative data have been defined. Recent investigations have explored the utility of HU values in assessing fracture risk, implant stability. The information provided by a simple HU measurement can alert the treating physician to decreased bone quality, which can be useful in both medically and surgically managing these patients [5].

Researchers studied that the density of bone has been related to the metastatic disease [6]. To estimate lower law density, we need to notice this relationship. However, little work has been done to evaluate the effect between tumors and bone density. Moreover, automatic parsing and segmentation of multiple organs and semantic navigation inside the body can help the clinician in efficiently obtaining an accurate diagnosis [7]. In this work, used the manually segmented low jaw bone. Further work will be consider about automatic segmentation based on whole body CT data.

Although our estimation uses only CT data, MRI data will be possible to use after converting MRI intensity to Hounsfield unit (HU) values. A method is needed to convert MRI data into CT like data. Some researchers investigated whether there is a relationship between MRI intensity and Hounsfield unit (HU) values [8].

Volume rendering is a flexible, accurate $3 \mathrm{D}$ imaging technique that can help the medical doctor more effectively interpret the large volumes of data generated by modern CT scanners [9, 10]. In this work, we used 3D slicer and interactive DICOM 3D viewer tools.

\section{Material AND Proposed Method (DMDCT1)}

\subsection{Material}

"CT mandible with few teeth" [11] image set that has some teeth in its lower jaw was used for the study.

* Size: this data is derived from 300 images that are parallel to the vertical axis of the Cartesian coordinate system called " $z$ " and an image is 400x400 pixels.

* Format: NRRD (Nearly Raw Raster Data)

Since open source CT data of mandible not found in the DICOM format, the NRRD format was converted to DICOM format using 3D slicer that mentioned above, and the lower jaw was extracted manually.

\subsection{Proposed Method (DMDCT1)}

\subsubsection{Algorithm}

The proposed method (called "DMDCT1") is described in Figure 2 as a block schema, and this section describes the main processes of DMDCT1 method more detailed.

* First, a start point of drilling and the diameter of the drill will be chosen by the doctor.

* Calculate the end slice of the drilling. In order to know the distance between slices that used to choose the slice that able to drill, to read header information of the DICOM formatted CT data and it is usually expressed in $\mathrm{mm}$.

* We will get points from the end slice of the drilling if their angle with a vertical axis is less than $10^{\circ}\left(15^{\circ}, 20^{\circ}\right.$, $25^{\circ}$ ).

* Assume that each of the selected points above is the center point of the top side and a point that doctor has chosen is center of the bottom side. Then, we will create cylinder its radius is equal to the radius of the drill.

* In each of the cylinders above, the morphological opening operation will be used to separate cortical bone (higher density than cancellous bone) and cancellous bone (also called trabecular bone).

* Lastly, we will select a cylinder that a number of points that have a value greater than 0 are maximum and an average of their value is greater than 400 .

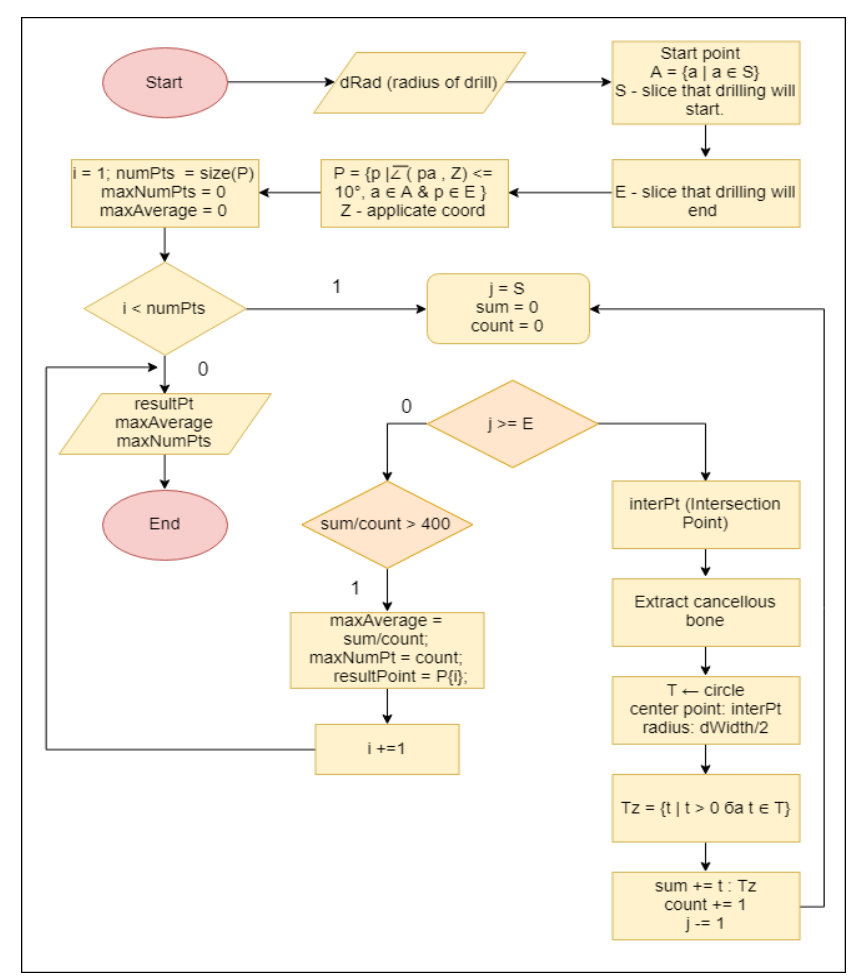

Fig. 1. Block schema of the presented algorithm. 


\subsubsection{Implementation}

We have implemented the algorithm with 2 phases. In the first phase, assumed that drill is like line, without considering the diameter of the drill. Then in the second phase, considered the diameter and length of the drill and represented the drill as a cylinder.

The following are pseudo codes of the function that play a major role in the implementation and toolbox pictures.

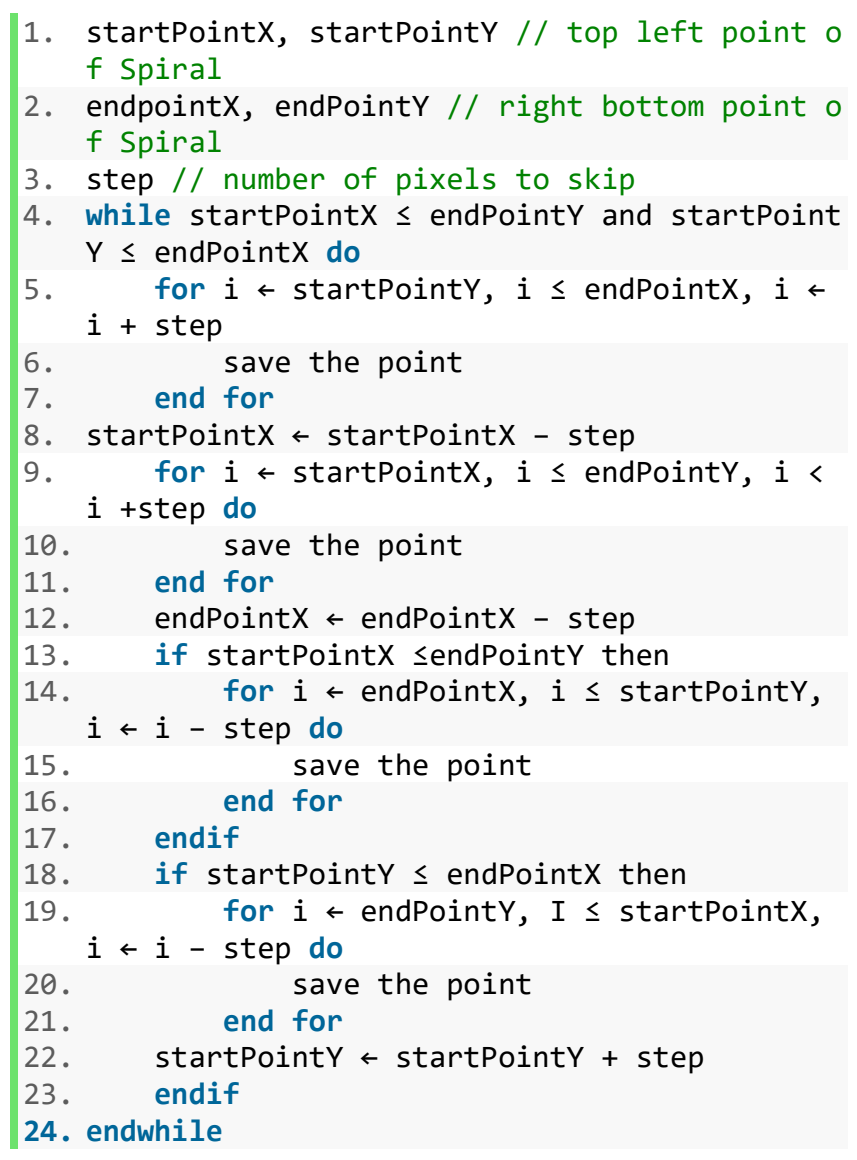

Fig. 2. Spiral loop for getting points to be used for calculation.

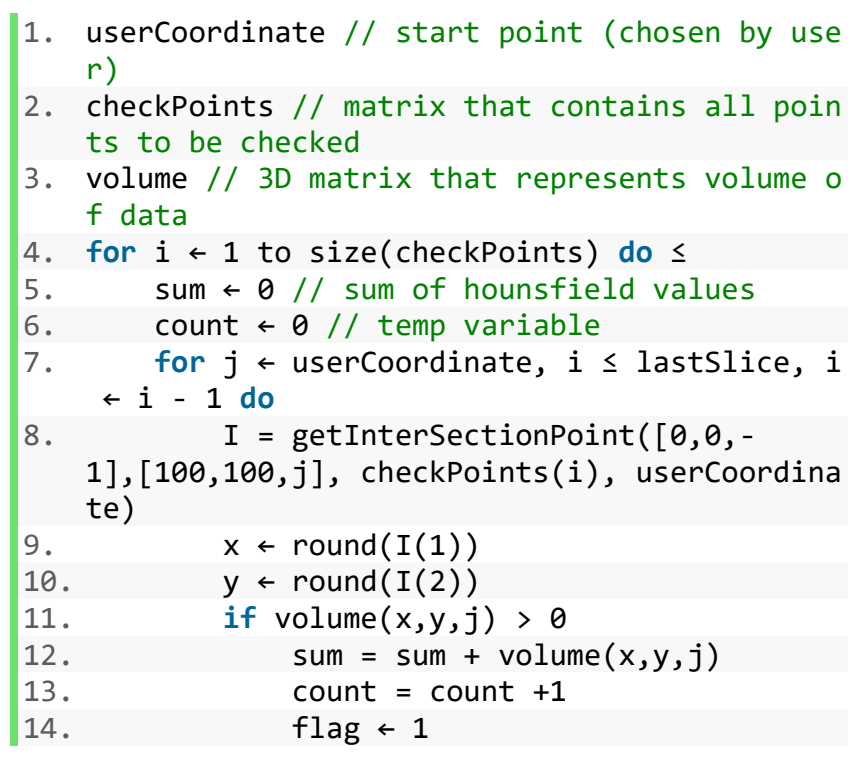

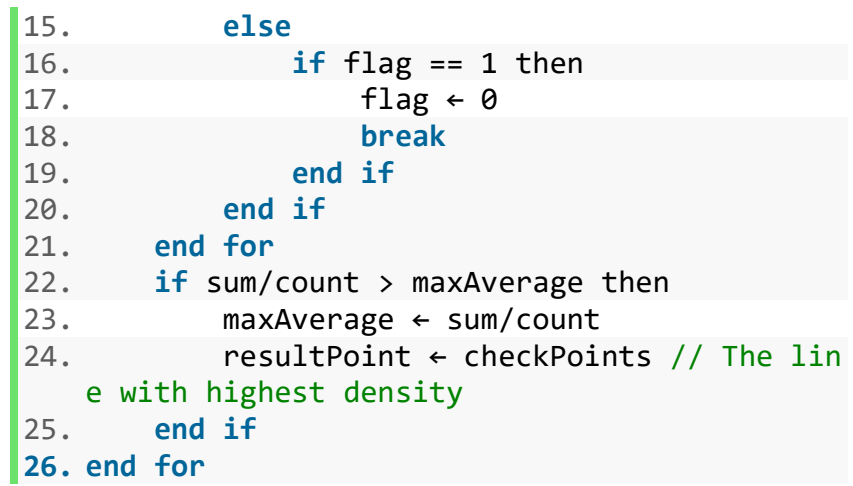

Fig. 3. Calculation of the highest density line.

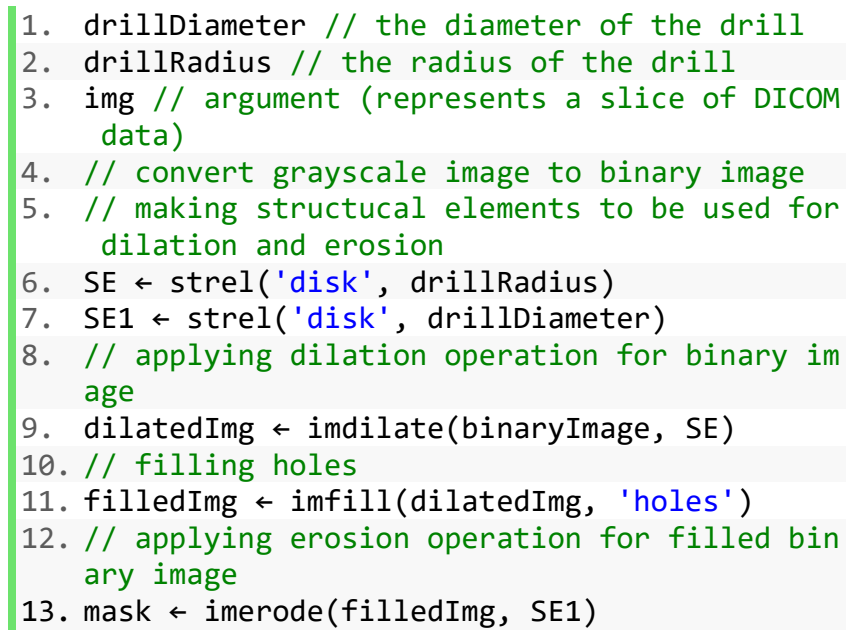

Fig. 4 Cancellous bone extraction.

\subsubsection{Improvement}

Reason for improvement: In the first phase, implementation of DMDCT1 was made without considering the diameter of the drill and it was too abstract. On the other hand, we had to make calculations involving the diameter of the drill and in order to make this, it was necessary to calculate in cylinders.

Problem: Experimental results were wrong because the value of the Hounsfield unit was high in cortical bone.

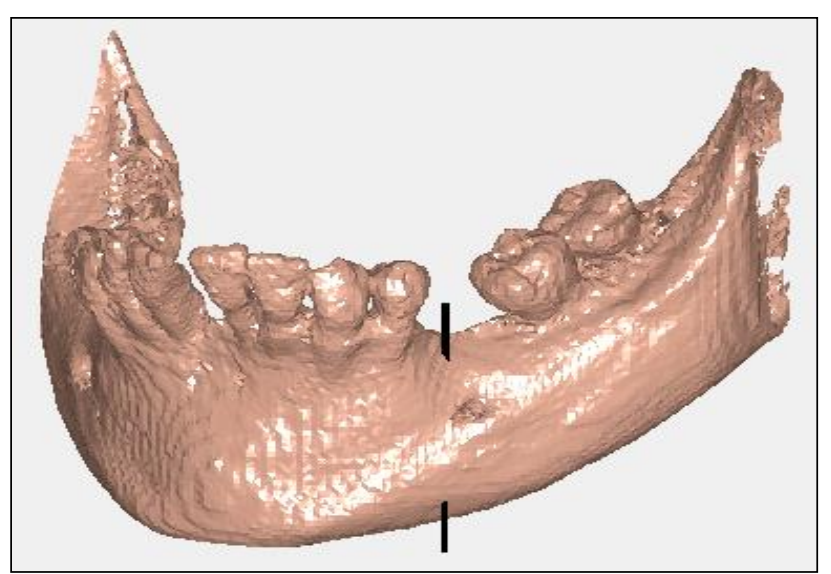

Fig. 5. Drawing of the highest density point. 


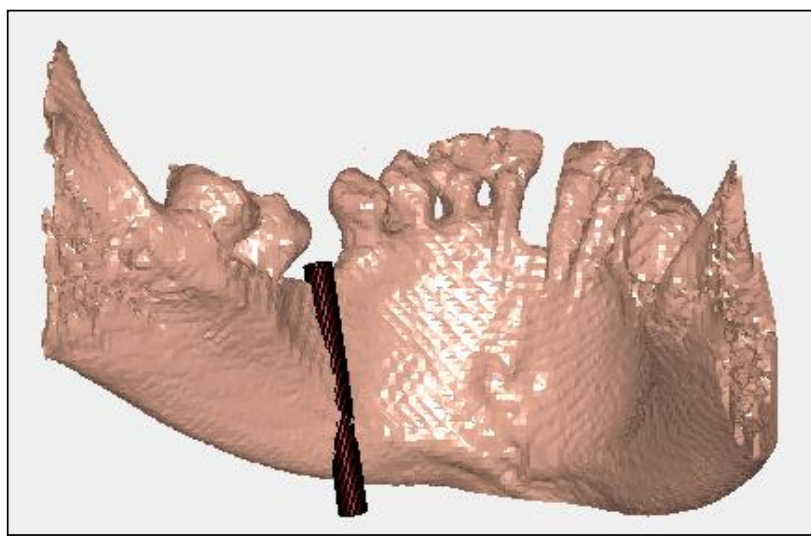

Fig. 6. Making the wrong result.

Solution: To extract cortical and cancellous bone and calculate for the cancellous bone only. To do this, the morphological closing operation will be performed on all CT slices that to be used for calculation.

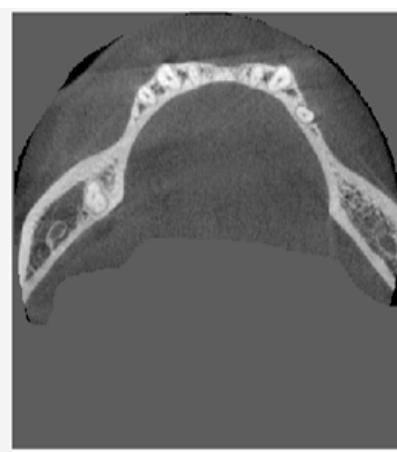

(a)

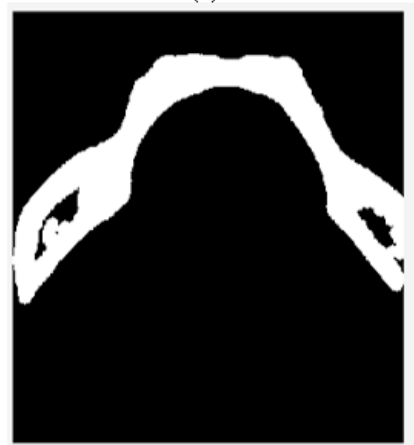

(c)

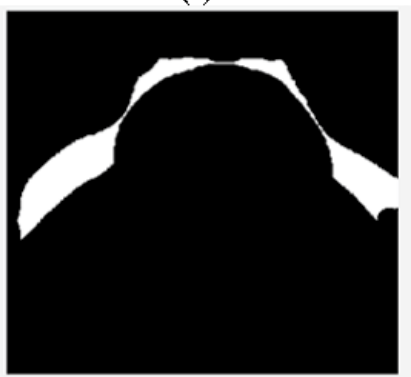

(e)

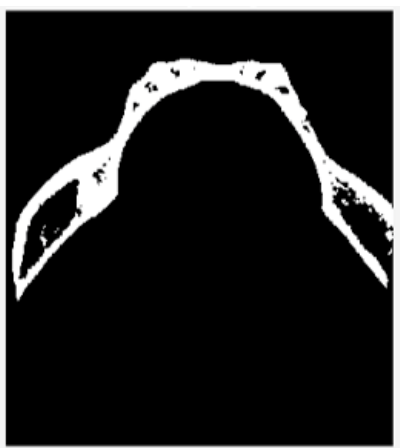

(b)

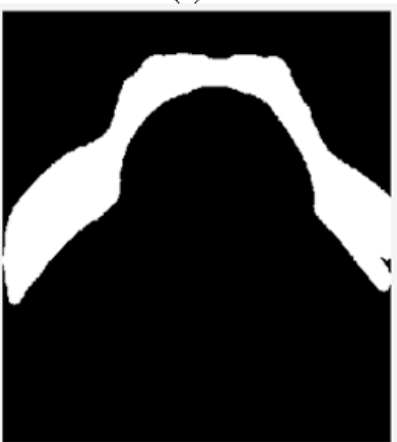

(d)

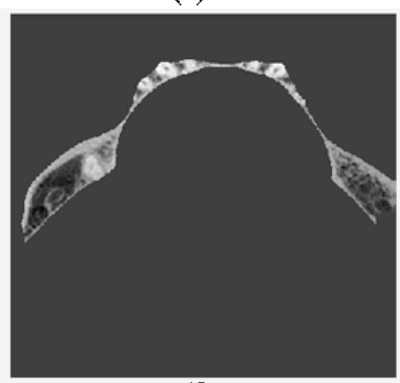

(f)
Fig. 7. Extracting cancellous bone. (a) Original grayscale image. (b) Binary image. (c) After applied dilation operator. (d) After filled holes. (e) After applied erosion operator. (f) Final grayscale image.

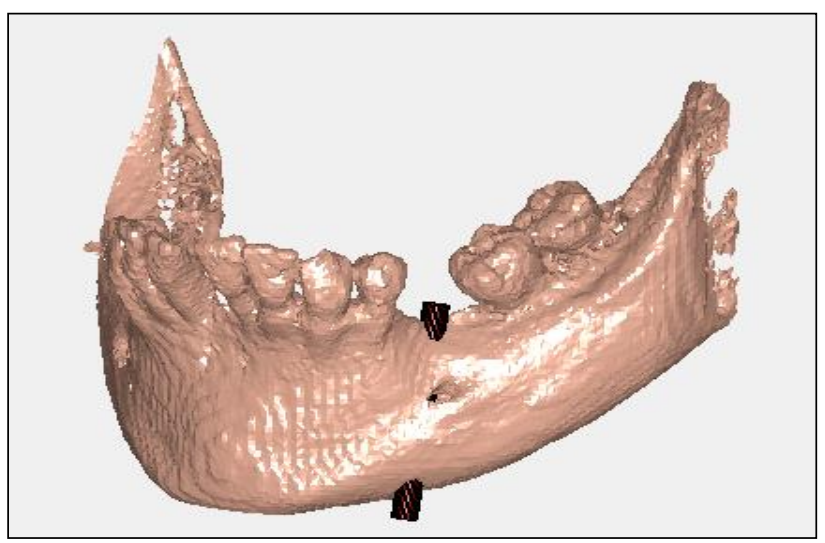

Fig. 8. After the correction of the wrong calculation using morphological closing operation (final result).

Note: In the above images, the cylinders and lines are shown more than bones in order to experimental results visible.

\subsubsection{Toolbox development}

This section will briefly consider the toolbox (Figure 9) and its appearance. In order to develop GUI-based toolbox, "Medical Image Reader and Viewer" tool developed by Josh Schaefferkoetter was used. This tool can read, view write, and save medical imaging data in DICOM format [12]. We had got permission from the developer of the tool to change the tool and use some parts that are needed to develop our own GUI-based toolbox for study.

The 3D volume viewer tool has been developed to demonstrate the experimental results of the study to the doctor in an understandable way. Lower jaw 3D volume model figures that have seen before were the result of this tool.

\subsection{Used Technologies}

* MATLAB [12]

$>$ GUIDE (GUI development environment)

$>$ Image Processing Toolbox

$>$ Interactive DICOM 3D Viewer

* 3D slicer
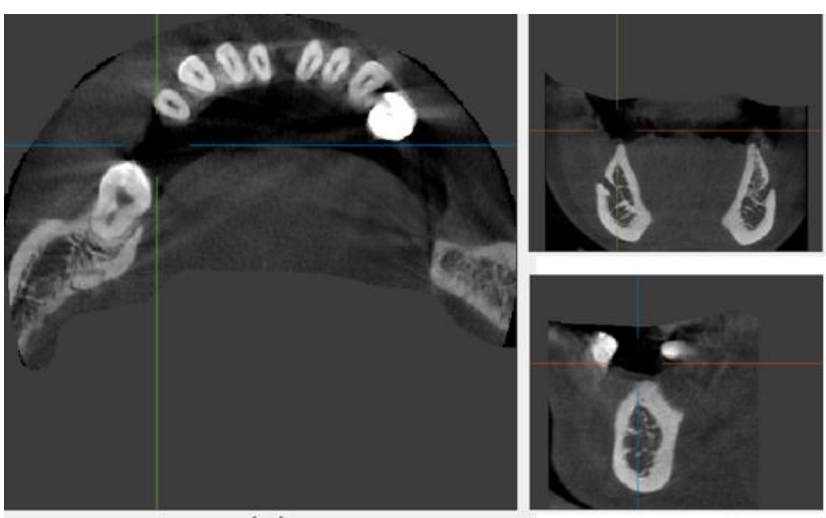

(b)

(a)

Fig. 9. Selecting a tooth to estimate the density (a toolbox that developed during the study). (a) Axial plane (X, Y). (b) Coronal plane (Y, Z). (c) Sagittal plane (X, Z). 


\section{EXPERIMENTAL RESULTS}

In order to evaluate experimental results, the following criteria were compared when the angle of a drill is less than or equal to $10^{\circ}, 15^{\circ}, 20^{\circ}, 25^{\circ}$ and the radius of a drill is 2 $\mathrm{mm}, 2.5 \mathrm{~mm}, 3 \mathrm{~mm}, 3.5 \mathrm{~mm}, 4 \mathrm{~mm}$ :
* Number of all checked cylinders

* Whether found cylinder that drilling is available

* Average Hounsfield value

* Time complexity (time to check all cylinders - sec)

Table 1 . When the angle between the drill and vertical axis is less than or equal to $10^{\circ}$.

\begin{tabular}{l|c|c|c|c|c}
\hline Drill radius (mm) & 2 & 2.5 & 3 & 3.5 & 4 \\
\hline Numbers of checked cylinders & 25 & 16 & 9 & 9 & 4 \\
\hline Whether found cylinder that drilling is available & $\checkmark$ & $\checkmark$ & $\checkmark$ & $\checkmark$ & $\checkmark$ \\
\hline Average Hounsfield value & 718.9 & 784.4 & 780.4 & 552.1 & 553.9 \\
\hline Time complexity & 39.7 & 22.6 & 11.2 & 10.4 & 9.3 \\
\hline
\end{tabular}

Table 2. When the angle between the drill and vertical axis is less than or equal to $15^{\circ}$.

\begin{tabular}{l|c|c|c|c|c}
\hline Drill radius (mm) & 2 & 2.5 & 3 & 3.5 & 4 \\
\hline Numbers of checked cylinders & 49 & 36 & 25 & 16 & 16 \\
\hline Whether found cylinder that drilling is available & $\checkmark$ & $\checkmark$ & $\checkmark$ & $\checkmark$ & $\checkmark$ \\
\hline Average Hounsfield value & 718.9 & 774.7 & 797.5 & 591.6 & 458.4 \\
\hline Time complexity & 72.4 & 52.1 & 33.6 & 23.1 & 27.8 \\
\hline
\end{tabular}

Table 3. When the angle between the drill and vertical axis is less than or equal to $20^{\circ}$.

\begin{tabular}{l|c|c|c|c|c}
\hline Drill radius (mm) & 2 & 2.5 & 3 & 3.5 & 4 \\
\hline Numbers of checked cylinders & 81 & 49 & 36 & 25 & 25 \\
\hline Whether found cylinder that drilling is available & $\checkmark$ & $\checkmark$ & $\checkmark$ & $\checkmark$ & $\checkmark$ \\
\hline Average Hounsfield value & 716.9 & 760.7 & 815.4 & 583.8 & 550.8 \\
\hline Time complexity & 128 & 75.3 & 48.9 & 39.9 & 38.1 \\
\hline
\end{tabular}

Table 4. When the angle between the drill and vertical axis is less than or equal to $20^{\circ}$.

\begin{tabular}{l|c|c|c|c|c}
\hline Drill radius (mm) & 2 & 2.5 & 3 & 3.5 & 4 \\
\hline Numbers of checked cylinders & 121 & 81 & 64 & 25 & 36 \\
\hline Whether found cylinder that drilling is available & $\checkmark$ & $\checkmark$ & $\checkmark$ & $\checkmark$ & $\checkmark$ \\
\hline Average Hounsfield value & 700.8 & 760.7 & 804.9 & 549.6 & 436.1 \\
\hline Time complexity & 188 & 127 & 92.8 & 80.6 & 57.1 \\
\hline
\end{tabular}




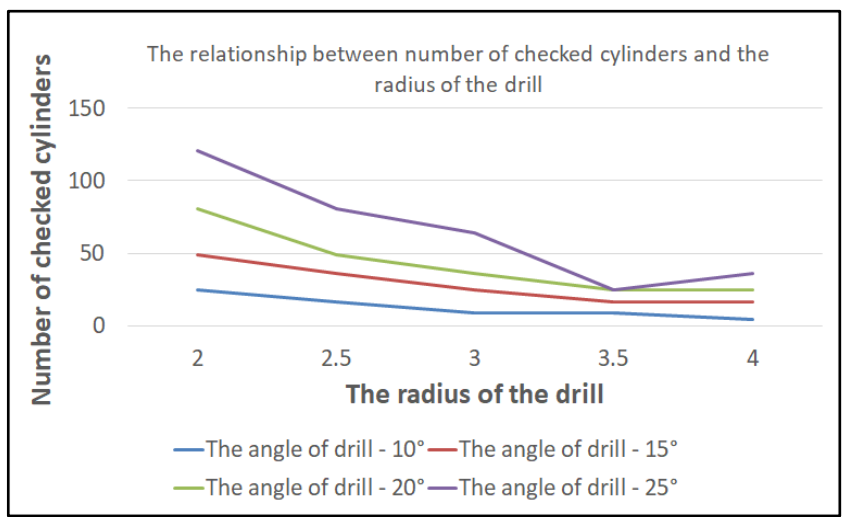

Fig. 10 The relationship between the time complexity and the radius of the drill.

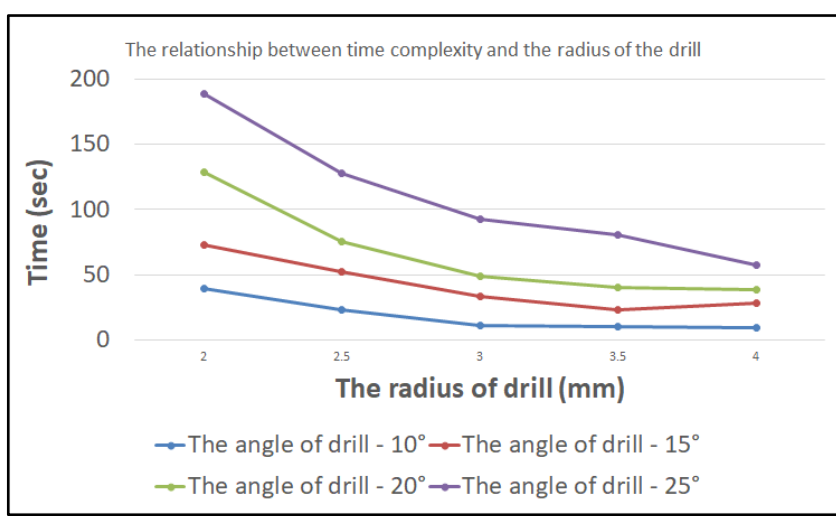

Fig. 11 The relationship between the number of checked cylinders and the radius of the drill.

\section{CONCLUSION}

As mentioned above, the proposed method (DMCT1) was implemented in two stages. As a result of these stages, the toolbox that developed after the first stage was approximately 9 times faster than the toolbox that developed after the second stage. However, considered drill as a line was too abstract and far from reality.

After the toolbox has been developed, the angle and diameter of the drill will be chosen differently and results have been evaluated by the four criteria that mentioned the experimental results section. The experimental results of the study show that when each time the angle between the drill and vertical axis of the Cartesian coordinate changed from $10^{\circ}$ to $25^{\circ}$ by $5^{\circ}$ interval, an average time of the calculation increased by 23 seconds, but cylinder that drilling is available was found, regardless of the angle of the drill. There was a positive correlation between the angle of the drill and time complexity but a negative correlation between the diameter of the drill and time complexity. Increasing the number of cylinders to be inspected led to high time complexity. Also, more than half of the time required to calculate was used for extracting cortical and cancellous bone using morphological image processing operations.

\section{Acknowledgement}

This work has been done within the framework of the project "Determination of Mandibular Density" supported by the Asian Research Center, Mongolia.

\section{REFERENCES}

[1] Dental Implants Market Size, Share \& Trends Analysis Report By Product (Titanium Implants, Zirconium Implants), By Region (North America, Europe, Asia Pacific, Latin America, MEA), And Segment Forecasts,2018-2024,

https://www.grandviewresearch.com/industryanalysis/dental-implants-market

[2] Success Rate of the EVL Evolution Implants (SERF): A Five-Year Longitudinal Multicenter Study, Journal of Oral Implantology, https://www.joionline.org/doi/full/10.1563/15481336\%282008\%2934\%5B283\%3ASROTEE\%5D2.0. CO\%3B2?=

[3] Hassan Mohajerani, Roozbeh Roozbayani, Shahram Therian, "The Risk Factors in Early Failure of Dental Implants: a Retrospective Study", Journal of Density, Vol.18(4), pp.298-303

[4] Dietmar Krappinger and et.al "Preoperative assessment of the cancellous bone mineral density of the proximal humerus using CT data." Skeletal radiology, Vol.41.3 (2012): 299-304.

[5] "Computer-assisted spine surgery: a technique for accurate transpedicular screw fixation using CT data and a 3-D optical localizer." Journal of image guided surgery Vol.1.1 (1995): 65-73.

[6] "Mechanical properties, density and quantitative CT scan data of trabecular bone with and without metastases." Journal of biomechanics, Vol.37.4 (2004): 523-530.

[7] "Hierarchical parsing and semantic navigation of full body CT data." Medical Imaging 2009: Image Processing. Vol. 7259. International Society for Optics and Photonics, 2009.

[8] "T1/T2*-weighted MRI provides clinically relevant pseudo-CT density data for the pelvic bones in MRIonly based radiotherapy treatment planning." Acta Oncologica Vol.52.3 (2013): 612-618.

[9] "Three-dimensional volume rendering of spiral CT data: theory and method." Radiographics, Vol.19.3 (1999): 745-764.

[10] "A new accurate and precise 3-D segmentation method for skeletal structures in volumetric CT data." IEEE transactions on medical imaging, Vol.22.5 (2003): 586-598.

[11] "CT mandible with few teeth image set", https://www.embodi3d.com/files/file/9196-ctmandibula-with-few-teeth

[12] "Medical Image Reader and Viewer Toolbox", https://www.mathworks.com/ https://www.mathworks.com/matlabcentral/fileexchan ge/53745-medical-image-reader-and-viewer 


\section{Authors}

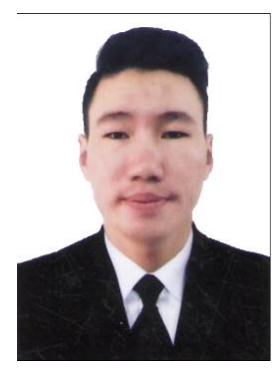

Ariunbold Jargalsaikhan received his BS degree in the Department of Information and Computer Sciences from the National University of Mongolia (NUM), Mongolia, in January 2019.

His research interests include image processing, pattern recognition, and computer vision.

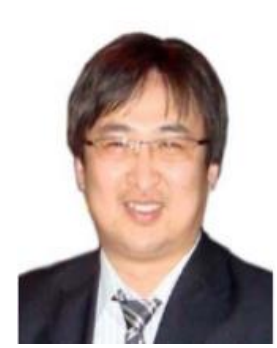

Nyamlkhagva Sengee received his M.Sc. (2008) and Ph.D. (2012) from the INJE University, Korea. $\mathrm{He}$ is an associate professor at the National University of Mongolia, since 2012. His research interests include image processing and analysis and image reconstruction.

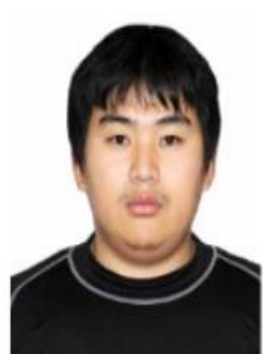

Berekjan Telue received his bachelor (2015) from the Mongolian University of Science and Technology. He is a master student at the National University of Mongolia, since 2016. His research interests include image processing and analysis and image reconstruction.

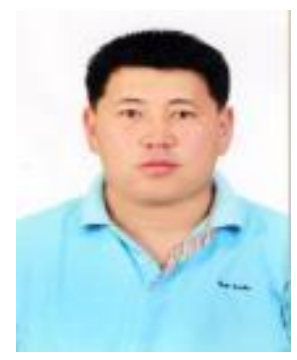

Sambuu Ochirkhuu received his bachelor (2005) and master (2007) from the Monos Medical University, Mongolia. His research interests include traditional medicine and traditional tuina threpy. 
